\title{
Phenotypic and Molecular Characterization of Candida dubliniensis Isolates from Clinical Specimens in Kuwait
}

\author{
Suhail Ahmad Eiman Mokaddas Noura Al-Sweih Zia U. Khan \\ Department of Microbiology, Faculty of Medicine, Kuwait University, Kuwait
}

\section{Key Words}

Candida dubliniensis · Candida albicans • Isolation • Molecular characterization $\cdot$ rDNA sequencing

\begin{abstract}
Objective: This study was carried out to characterize Candida dubliniensis using phenotypic and molecular methods and to determine the occurrence of $C$. dubliniensis in clinical specimens in Kuwait. Materials and Methods: A total of 880 clinical specimens for isolation of fungi were processed according to standard procedures. Of these, 390 germ-tube-positive clinical isolates of Candida species were examined for rough colonies with hyphal fringes and chlamydospore production on simplified sunflower seed agar for their presumptive phenotypic identification as $C$. dubliniensis. The identification of $C$. dubliniensis isolates was further confirmed by the Vitek 2 yeast identification system, semi-nested (sn) PCR amplification of high-copy rDNA and direct DNA sequencing of the internally transcribed spacer 2 (ITS2) region. Results: Of the 390 isolates of Candida species investigated, 12 were identified as $C$. dubliniensis, giving an overall occurrence of $3 \%$. All the $C$. dubliniensis isolates formed rough colonies with hyphal fringes and abundant chlamydospores on sunflower seed agar, did not assimilate trehalose, lactate and $\alpha$-methyl- $D$-glucoside, and were isolated from human immunodeficiency virus (HIV)-negative patients. Four $C$. dubliniensis isolates utilized $D$-xylose. The species-specific primer derived from the ITS2 sequence of $C$. dubliniensis and used
\end{abstract}

together with the panfungal reverse primer in the reamplification step of the snPCR specifically amplified rDNA from reference and clinical $C$. dubliniensis isolates and not from $C$. albicans or other Candida species. The identity of two representative isolates was confirmed by DNA sequencing of the ITS2 region. Conclusions: The identity of 12 C. dubliniensis isolates was first established by phenotypic characteristics and then by snPCR using species-specific primers derived from ITS2 sequences. The recovery of $C$. dubliniensis from HIV-negative patients from Kuwait reinforces the existing view that this novel yeast species has a worldwide distribution and its occurrence is not restricted to any particular immunocompromised population.

Copyright $\odot 2005$ S. Karger AG, Basel

\section{Introduction}

Candida dubliniensis is a newly recognized species that is genotypically distinct from C. albicans but shares several phenotypic characteristics with the latter species [1]. It occurs as a minor constituent of the human oral microflora [2]. In the past, C. dubliniensis isolates have been misidentified as C. albicans, since both species are germ tube positive and produce chlamydospores on cornmeal Tween 80 agar [1-3]. Although initially C. dubliniensis was believed to be associated with oropharyngeal candidiasis in human immunodeficiency virus (HIV)-infected patients $[4,5]$, recent reports suggest that this species has a much wider epidemiologic significance [2]. The species

\section{KARGER \\ Fax +4161306 1234 E-Mail karger@karger.ch} www.karger.com
C 2005 S. Karger AG, Basel

1011-7571/05/0147-0077\$22.00/0

Accessible online at:

www.karger.com/mpp
Prof. Zia U. Khan

Department of Microbiology, Faculty of Medicine, Kuwait University PO Box 24923, 13110 Safat (Kuwait)

Tel. +965 531 2300, ext. 6505, Fax +965 5318454, +965 5332719

E-Mail ziauddin@hsc.edu.kw 
has been isolated from a variety of clinical specimens and anatomic sites of HIV-negative as well as HIV-positive individuals with and without symptoms of oral candidiasis [4-8].

Given the growing importance of $C$. dubliniensis as an opportunistic pathogen [7-9], development of rapid methods to discriminate $C$. dubliniensis from $C$. albicans is highly desirable. Recently, several seed-based and nonseed-based media have been described to discriminate C. dubliniensis from C. albicans on the basis of colony characteristics and chlamydospores production [10-15]. Rapid identification of clinical C. dubliniensis isolates has also been described by the use of specific primers in PCR $[16,17]$.

In this study, we report isolation and identification of 12 isolates of $C$. dubliniensis from non-HIV-infected patients from Kuwait during a 1-year period. The identification of the $C$. dubliniensis isolates was performed by phenotypic characteristics and by a semi-nested PCR (snPCR) using panfungal and C. dubliniensis-specific primers as described in the previous studies [18-20].

\section{Materials and Methods}

\section{Isolation and Phenotypic Characterization}

A total of 880 clinical specimens (vaginal swab: 410; urine: 123; bronchoalveolar lavage: 85; sputum: 81 ; cerebrospinal fluid: 46; blood culture: 40; tracheal secretion: 36 ; peritoneal and gastric fluid: 21 ; throat swab: 14; wound swab: 7; catheter tip: 7; tissue biopsies: 6 , and bile fluid: 4) for the isolation of fungi received at the Mycology Laboratory during January to December 2003 were processed according to standard procedures as previously described [21]. All the yeast isolates $(n=452)$ were streaked on Sabouraud glucose agar (glucose $15 \mathrm{~g}$, peptone $10 \mathrm{~g}$ and agar $15 \mathrm{~g}$ in 1 liter distilled water, $\mathrm{pH}$ 6.8) supplemented with chloramphenicol (50 mg/l) and checked for purity. The inoculum from the isolated yeast colonies was tested for germ tube formation in serum at $37^{\circ} \mathrm{C}$. The germ tube-positive isolates (390) were examined for colonial and microscopic characteristics on simplified sunflower seed agar as previously described [10]. Isolates producing rough colonies with hyphal fringes and chlamydospores were provisionally identified as C. dubliniensis. They were further identified by their inability to assimilate trehalose and $\alpha$-methyl- $D$-glucoside in the Vitek 2 yeast identification system (BioMerieux, Marey l'Etoile, France). Isolates were also tested for tolerance to $6.5 \%$ sodium chloride to distinguish C. dubliniensis from C. albicans [22].

\section{Molecular Characterization}

C. dubliniensis (CD 36), C. albicans (ATCC 76615), C. parapsilosis (ATCC 10233), C. tropicalis (ATCC 750), C. glabrata (ATCC 15545), C. krusei (clinical isolate) and C. lusitaniae (clinical isolate) were used as reference Candida species for snPCR amplification of rDNA. In addition, DNA extracted from Aspergillus fumigatus (ATCC 13073), A. terreus (CBS 118.27), Fusarium solani
(ATCC 36031), Trichosporon asahii CBS 2530, Cryptococcus neoformans (ATCC 90112), Toxoplasma gondii (clinical isolate), human cells, Mycobacterium tuberculosis $\mathrm{H}_{37} \mathrm{Rv}$ and Escherichia coli BL-21 was also included in the study.

\section{snPCR Amplification Method}

The genomic DNA was extracted from the grown cultures of the isolates as previously described $[18,19]$. The sequences of the universal forward and reverse fungal primers, capable of amplifying the $3^{\prime}$ end of $5.8 \mathrm{~S}$ and $5^{\prime}$ end of $28 \mathrm{~S}$ rDNA including the internally transcribed spacer 2 (ITS2) region and the species-specific oligonucleotide primer, derived from the ITS2 region of $C$. dubliniensis, have already been described [18-20]. Amplification with universal fungal primers and species-specific primer together with universal fungal reverse primer in the first round and snPCR from $C . d u b l i-$ niensis CD 36 should yield DNA fragments of $350 \mathrm{bp}$ and $105 \mathrm{bp}$, respectively [20]. Amplification of target DNA was carried out in thin-walled $0.2-\mathrm{ml}$ PCR tubes in a total volume of $50 \mu$ l containing 1× AmpliTaq PCR buffer I, $1 \mathrm{U}$ AmpliTaq DNA polymerase, 10 pmol each of CTSF and CTSR primers, $1 \mu$ of DNA and $0.1 \mathrm{~m} M$ of each dNTP. After amplification in the first step, $1 \mu \mathrm{l}$ of the product was further amplified using the initial reverse primer (CTSR) and C. dubliniensis-specific forward primer. For snPCR, the reaction mixture consisted of $1 \times$ AmpliTaq PCR buffer I, $1 \mathrm{U}$ AmpliTaq DNA polymerase, 5 pmol of CTSR together with 5 pmol of CDDET; $1 \mu \mathrm{l}$ of the first PCR product and $0.1 \mathrm{~m} M$ of each dNTP. PCR cycling (total 30 cycles) was carried out in a PerkinElmer cycler (GeneAmp PCR system 2400) under the following conditions: denaturation at $94^{\circ} \mathrm{C}$ for $1 \mathrm{~min}$, annealing at $60^{\circ} \mathrm{C}$ for $30 \mathrm{~s}$ and extension at $72^{\circ} \mathrm{C}$ for $1 \mathrm{~min}$. An initial denaturation step at $95^{\circ} \mathrm{C}$ for $3 \mathrm{~min}$ and a final extension step at $72^{\circ} \mathrm{C}$ for $10 \mathrm{~min}$ were also included. To detect amplified DNA fragments, agarose gel electrophoresis was performed as previously described [18]. The gels were exposed to UV light and photographed. Appropriate negative controls were included in each test run, including controls omitting the DNA template during PCR assays.

The DNA sequence of the ITS2 region of two representative clinical C. dubliniensis isolates from Kuwait (MF 1042 and MF 122) was determined by amplification of the ITS2 region with universal fungal primers. The sequencing reactions with amplified DNA as template and forward or reverse panfungal primer were performed as described elsewhere [23]. Briefly, the column-purified PCR-amplified fragment obtained with the panfungal primer pair was used as template in sequencing reactions using the cycle DNA sequencing kit (DTCS CEQ2000, Beckman Coulter). The reaction mixtures in a final volume of $20 \mu$ l contained eluted DNA, 5 pmol of CTSF or CTSR primer and $8.0 \mu$ l of the premix reaction components supplied in the kit. The cycling parameters for the sequencing reactions included an initial denaturation at $95^{\circ} \mathrm{C}$ for $5 \mathrm{~min}$ followed by 30 cycles of $1 \mathrm{~min}$ at $94^{\circ} \mathrm{C}, 30 \mathrm{~s}$ at $55^{\circ} \mathrm{C}$ and $2 \mathrm{~min}$ at $60^{\circ} \mathrm{C}$. The reaction products were precipitated and loaded on the sequencer as directed by the manufacturer of the automated DNA sequencer (Beckman Coulter Model CEQ8000).

\section{Antifungal Susceptibility Testing}

Antifungal susceptibility of the 12 C. dubliniensis isolates was determined against amphotericin B, fluconazole and voriconazole by E-test (AB BIODISK, Solna, Sweden). Each C. dubliniensis isolate was freshly subcultured and checked for purity. Five isolated colonies were uniformly suspended in sterile saline solution and 
Fig. 1. a Simplified sunflower seed agar showing white rough colonies with hyphal fringes of $C$. dubliniensis, white smooth colonies of $C$. albicans and dark colonies of $C$. neoformans cultured from experimentally seeded bronchoalveolar lavage sample. b Slide culture on simplified sunflower seed agar showing chlamydospores of $C$. dubliniensis (magnification $\times 400$ ).
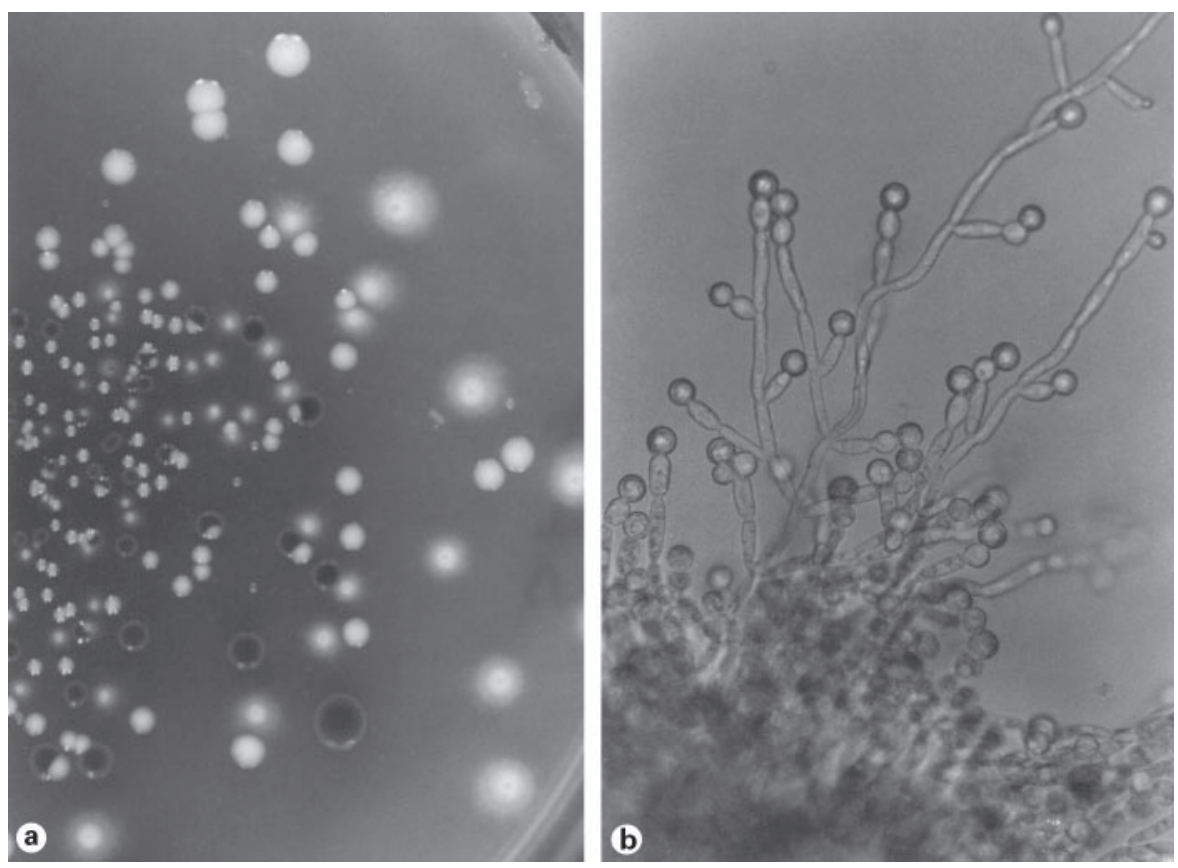

turbidity was adjusted to 0.5 on the McFarland standard scale. This inoculum was then streaked onto the Sabouraud dextrose agar Petri plates (150 $\mathrm{mm}$ diameter) and allowed to dry at room temperature for 10-15 min before the application of the E-test strips. The RPMI-1640 agar supplemented with 2\% glucose and buffered with MOPS, 0.165 M, pH 7.0, was used for antifungal susceptibility testing (National Committee for Clinical Laboratory Standards, NCCLS, M-27A) [24]. The plates were incubated in an incubator at $35^{\circ} \mathrm{C}$ for $1-2$ days and minimal inhibitory concentrations (MICs) were read after $24-48 \mathrm{~h}$ of incubation. The point where inhibition ellipses intercepted the scale on the antifungal strip was taken as MIC for each test isolate. Complete inhibition (100\%) of growth for amphotericin B and marked decrease in growth intensity for fluconazole and voriconazole were observed $[25,26]$.

\section{Results}

Of the 390 germ tube-positive isolates of Candida species, 12 were identified as $C$. dubliniensis based on colonial and microscopic characteristics on simplified sunflower seed agar medium. All the 12 C. dubliniensis isolates formed rough colonies with hyphal fringes and abundant chlamydospores on this medium after $48 \mathrm{~h}$ of incubation at $30^{\circ} \mathrm{C}$ (fig. 1a, b), while none of the remaining 378 isolates exhibited these characteristics. The results of phenotypic and genotypic characterization and the antifungal susceptibility profile are presented in tables 1,2 and figures $1-3$. Among the 12 isolates of C. dubliniensis, 9 were recovered from the respiratory tract and 1 each from urine, vaginal swab and catheter tip. All the 12 C. dubliniensis isolates originated from HIV-negative patients and none of these patients had systemic Candida infection. None of the 12 C. dubliniensis isolates assimilated $\alpha$-methyl- $D$-glucoside, lactate and trehalose after $24 \mathrm{~h}$ of incubation in the Vitek 2 yeast identification system. Four $C$. dubliniensis isolates assimilated $D$-xylose. The growth of $C$. dubliniensis isolates was inhibited in the presence of $6.5 \%$ sodium chloride (table 1).

The results of the E-test performed on the $12 C . d u b$ liniensis isolates are presented in table 2. The E-test MIC ranges for the three antifungal agents were as follows: amphotericin B $0.002-0.038 \mu \mathrm{g} / \mathrm{ml}$, fluconazole 0.094 $0.75 \mu \mathrm{g} / \mathrm{ml}$, and voriconazole $0.003-0.012 \mu \mathrm{g} / \mathrm{ml}$. None of $C$. dubliniensis isolate was resistant to any of the three antifungal agents tested.

The PCR amplification of rDNA from the 7 Candida species, i.e., C. albicans, C. dubliniensis, C. parapsilosis, C. tropicalis, C. glabrata, C. krusei and C. lusitaniae, using universal fungal primers (CTSF and CTSR) resulted in amplification of DNA fragments of expected sizes (fig. 2a). Similar results were obtained when the genomic DNA prepared from A. fumigatus, F. solani, T. asahii and C. neoformans was used as template (data not shown). Reamplification of the product of the first PCR with CTSR and CDDET resulted in specific amplification of a single DNA fragment of the expected size from the reference strain of $C$. dubliniensis only and not from the 6 


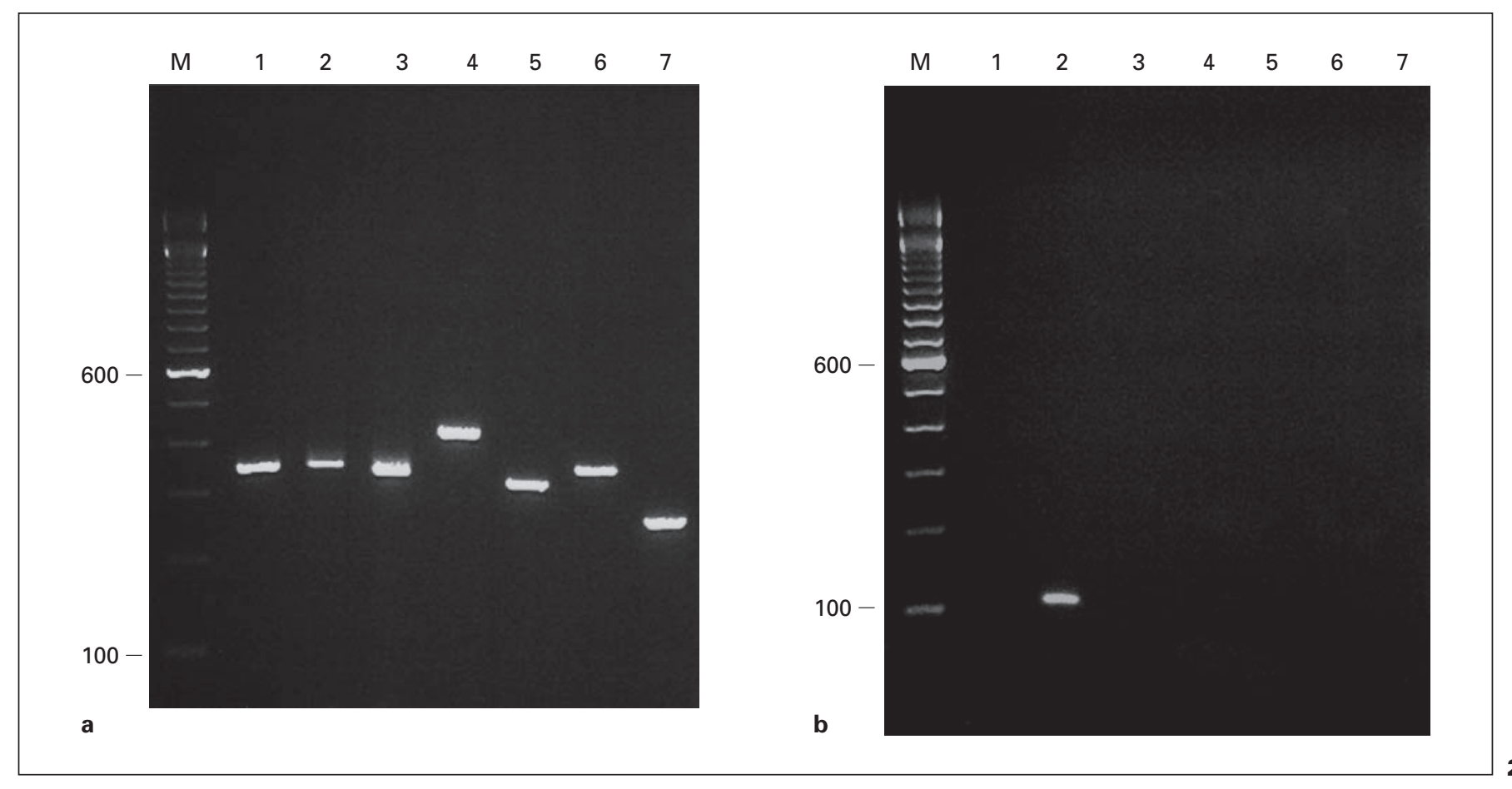

Fig. 2. Agarose gel electrophoresis of the product of the first round of PCR amplification using universal fungal primers (CTSF and CTSR) (a) and snPCR amplification using CTSR and CDDET primers (b) of genomic DNA from $C$. albicans (lane 1), $C$. dubliniensis (lane 2), C. tropicalis (lane 3), C. glabrata (lane 4), C. parapsilosis (lane 5), C. krusei (lane 6) and C. lusitaniae (lane 7). Lane $\mathrm{M}$ is a 100-bp DNA ladder and the positions of migration of 100- and 600-bp fragments are marked.

Fig. 3. Agarose gel electrophoresis of the product of the first round of PCR amplification using universal fungal primers (CTSF and CTSR) (a) and snPCR amplification using CTSR and CDDET primers (b) of genomic DNA from the 4 clinical C. dubliniensis isolates MF 1042, MF 41, MF 48 and MF 122 (lanes 1-4, respectively). Other details are the same as in the legend of

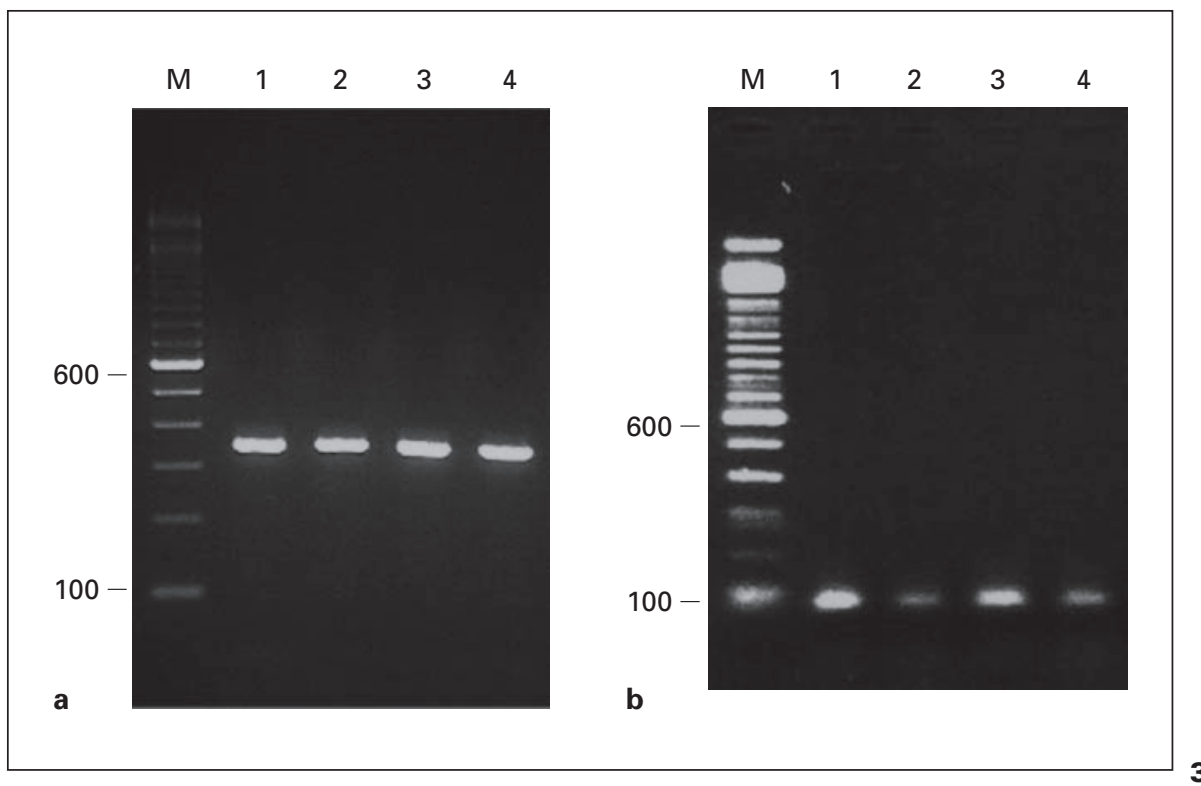
figure 2 .

other Candida species, i.e., C. albicans, C. parapsilosis, C. tropicalis, C. glabrata, C. krusei, and C. lusitaniae (fig. 2b), or other fungal pathogens (e.g. A. fumigatus, F. solani, T. asahii and C. neoformans; data not shown). No amplification was detected with genomic DNA from nonfungal organisms in the first as well as the second step of snPCR (data not shown).
The PCR amplification of rDNA from all the 12 clinical isolates of $C$. dubliniensis recovered from Kuwait, using CTSF and CTSR primers, resulted in specific amplification of a single DNA fragment of the expected size (the data from four isolates are shown in figure $2 \mathrm{a}$, lanes 1-4) from each isolate. Further, reamplification of the products of the first PCR with CTSR and CDDET resulted again in specific amplification of a single DNA 
Table 1. Phenotypic characteristics of C. dubliniensis isolates recovered from clinical specimens in Kuwait

\begin{tabular}{|c|c|c|c|c|c|c|c|c|}
\hline \multirow[t]{2}{*}{$\begin{array}{l}\text { Isolate } \\
\text { No. }\end{array}$} & \multirow[t]{2}{*}{ Source } & \multicolumn{2}{|c|}{$\begin{array}{l}\text { Characteristics } \\
\text { on SSA }\end{array}$} & \multicolumn{5}{|c|}{ Growth at or on } \\
\hline & & colony & $\begin{array}{l}\text { chlamydo- } \\
\text { spores }\end{array}$ & xylose & MDG & $\begin{array}{l}\text { lac- } \\
\text { tate }\end{array}$ & $\begin{array}{l}\text { treha- } \\
\text { lose }\end{array}$ & $\begin{array}{l}6.5 \% \\
\mathrm{NaCl}\end{array}$ \\
\hline MF 1042 & urine & rough & + & - & - & - & - & - \\
\hline MF 41 & sputum & rough & + & - & - & - & - & - \\
\hline MF 48 & sputum & rough & + & - & - & - & - & - \\
\hline MF 122 & sputum & rough & + & - & - & - & - & - \\
\hline S 4 & sputum & rough & + & - & - & - & - & - \\
\hline S 12 & vaginal swab & rough & + & + & - & - & - & - \\
\hline S 59 & sputum & rough & + & + & - & - & - & - \\
\hline MF 272 & sputum & rough & + & - & - & - & - & - \\
\hline MF 311 & catheter tip & rough & + & - & - & - & - & - \\
\hline MF 426 & BAL & rough & + & + & - & - & - & - \\
\hline MF 460 & tracheal secretion & rough & + & + & - & - & - & - \\
\hline MF 523 & throat swab & rough & + & - & - & - & - & - \\
\hline
\end{tabular}

$\mathrm{SSA}=$ Sunflower seed agar; $\mathrm{BAL}=$ bronchoalveolar lavage $\mathrm{MDG}=\alpha$-methyl- $D$-glucoside.
Table 2. Antifungal susceptibility data of 12 isolates of C. dubliniensis by E-test

\begin{tabular}{lllll}
\hline \multirow{2}{*}{$\begin{array}{l}\text { Antifungal } \\
\text { agents }\end{array}$} & \multicolumn{4}{l}{ Antifungal susceptibility, $\mu \mathrm{g} / \mathrm{ml}$} \\
\cline { 2 - 5 } & range & $\mathrm{MIC}_{50}$ & $\mathrm{MIC}_{90}$ & \% resistance \\
\hline Amphotericin B & $0.002-0.38$ & 0.023 & 0.047 & 0 \\
Fluconazole & $0.094-0.75$ & 0.19 & 0.25 & 0 \\
Voriconazole & $0.003-0.012$ & 0.006 & 0.012 & 0 \\
\hline
\end{tabular}

fragment (the data from the same four isolates are shown in figure $2 b$, lanes $1-4$ ) of the same size as that obtained from the reference strain of $C$. dubliniensis. Finally, the identity of two of the representative clinical isolates (MF 1042 and MF 122) as C. dubliniensis was confirmed by DNA sequencing of the ITS2 region of rDNA. The DNA sequence of the ITS2 region of these isolates completely matched only with the $C$. dubliniensis strain CD 36 but not with $C$. albicans or any other Candida species. These data confirmed the isolates recovered from HIV-negative patients in Kuwait as C. dubliniensis.

\section{Discussion}

This study reports recovery of 12 isolates of $C$. dubliniensis from a range of clinical samples and originating from patients with different underlying conditions within a span of 1 year. The identity of the isolates has been unequivocally established as $C$. dubliniensis by known phenotypic and molecular characteristics. In most of the published studies, differences in the assimilation of xylose, trehalose, and $\alpha$-methyl- $D$-glucoside have been used to separate $C$. dubliniensis from C. albicans $[1,27,28]$. However, some recent reports suggest that assimilation characteristics of carbohydrates between the 2 species are not as discriminatory as initially reported and may vary depending upon the assimilation method employed [29, 30]. The ability of 4 of our $C$. dubliniensis isolates to assimilate $D$-xylose within $24 \mathrm{~h}$ with the Vitek 2 yeast identification system also suggests the inconsistency of the assimilation profiles in distinguishing these two closely related Candida species. Using a novel automated assimilation system, Kurzai et al. [30] reported that $C$. dubliniensis isolates were able to utilize $D$-xylose and trehalose. In another study [29], while the 24-hour profiles obtained with the Vitek 2 yeast identification system showed that $\alpha$-methyl- $D$-glucoside, $D$-xylose and glycerol could be used to separate $C$. dubliniensis from $C$. albicans, at $48 \mathrm{~h}$ only $D$-xylose (6 vs. $95 \%$ ) was useful for separating the two species. Following comparative evaluation of different commercial systems, these investigators [29] concluded that the assimilation of $\alpha$-methyl- $D$-glucoside, $D$-xylose and trehalose was most useful for the differentiation of the two species by the majority of commercial systems. Moreover, it was the rapidity of the assimilation achieved with the commercial systems that allowed the differentiation of $C$. dubliniensis from C. albicans. It is apparent 
that while most of the $C$. dubliniensis isolates can be distinguished by carbohydrate assimilation results, some strains may give inconsistent results justifying application of PCR-based methods for unequivocal identification [29-32].

As our understanding on the occurrence and pathogenesis of this novel species is increasing, it is becoming apparent that this pathogen has the potential to cause a wide spectrum of clinical conditions both in HIV-infected and non-HIV-infected patients such as those receiving chemotherapy for cancer, organ transplant recipients or patients with other underlying conditions $[2,4,6,8,33,34]$. A review of published reports suggests that $C$. dubliniensis is widely distributed in the population but forms only a minor constituent of the microbial flora in HIV-negative individuals $[1,2,32]$. Although the species may be found at any anatomic site, it probably exhibits greater propensity to colonize oral mucosa particularly of HIVinfected patients $[2,4,5,34,35]$. In our study, none of the $C$. dubliniensis isolates came from HIV-infected patients, but as many as 9 of the $12(75 \%)$ were isolated from respiratory tract specimens (table 1 ). The other three isolates were recovered from catheter tip, urine and vaginal specimens. Other studies have also reported the isolation of $C$. dubliniensis from a wide range of clinical specimens including bronchoalveolar lavage, urine and catheter tip $[37,38]$. In a study of the Irish population of normal healthy individuals, only $3.5 \%$ were found to carry $C . d u b$ liniensis in the oral cavity, while the prevalence of this species in the vagina was found to be even lower (2.7\%) [34]. In contrast, $26 \%$ of HIV-infected and 32\% of AIDS patients with symptoms of oropharyngeal candidiasis yielded C. dubliniensis, whereas in patients without symptoms of oropharyngeal candidiasis the colonization levels were 18 and 25\%, respectively [35]. Like in other regions of the world [2,32], occurrence of $C$. dubliniensis has also been recognized in the Middle East [20, 33, 34, 36-38].
Fotedar and Al-Hedaithy [38] studied 823 germ tubepositive and chlamydospore-positive $C$. albicans isolates at the University Hospital in Riyadh, Saudi Arabia. On reexamination, 27 (3.3\%) of them were identified as $C$.dubliniensis. The prevalence of $C$. dubliniensis amongst germ tube-positive isolates was almost similar $(3 \%)$ in the present study.

It has been suggested that the recent emergence of C. dubliniensis as a human pathogen may have resulted due to the widespread use of azole antifungal therapy in HIV-infected patients, in a manner similar to that suggested for C. glabrata [1, 2]. However, this assumption may not be correct since it has been found that the great majority of $C$. dubliniensis isolates are inherently susceptible to commonly used antifungal agents [5, 39]. Similarly, all the 12 C. dubliniensis isolates of this study were susceptible to fluconazole and voriconazole and also to amphotericin B.

\section{Conclusions}

We have described the isolation and identification of 12 isolates of $C$. dubliniensis recovered mostly from the respiratory tract of HIV-negative patients in Kuwait during a 1-year period. As our understanding of the epidemiology of C. dubliniensis is increasing, it is becoming apparent that this pathogen can colonize different anatomic sites with a potential to cause superficial or invasive disease.

\section{Acknowledgements}

We thank Rachel Chandy and Da'ad Farhat for excellent technical assistance. This work was supported by Kuwait University Research Administration grant No. MI 118.

\section{References}

-1 Sullivan D, Coleman DC: Candida dubliniensis: Characteristics and identification. J Clin Microbiol 1998;36:329-334.

$\checkmark 2$ Sullivan DJ, Moran GP, Pinjon E, Al-Mosaid A, Stokes C, Vaughan C, Coleman DC: Comparison of the epidemiology, drug resistance mechanisms, and virulence of Candida dubliniensis and Candida albicans. FEMS Yeast Res 2004;4:369-376.
- 3 Odds FC, Van Nuffel L, Dams G: Prevalence of Candida dubliniensis isolates in a yeast stock collection. J Clin Microbiol 1998;36:28692873.

4 Jabra-Rizk MA, Falkler WA Jr, Merz WG, Baqui AA, Kelly JI, Meiller TF: Retrospective identification and characterization of Candida dubliniensis isolates among Candida albicans clinical laboratory isolates from human immunodeficiency virus (HIV)-infected and nonHIV-infected individuals. J Clin Microbiol 2000;38:2423-2426.

\footnotetext{
5 Moran GP, Sullivan DJ, Henman MC, McCreary CE, Harrington B, Shanley DB, Coleman DC: Antifungal drug susceptibilities of oral Candida dubliniensis isolates from human immunodeficiency virus (HIV)-infected and non-HIV-infected subjects and generation of stable fluconazole-resistant derivatives in vitro. Antimicrob Agents Chemother 1997;41:617-623.
} 
-6 Brandt ME, Harrison LH, Pass M, Sofair AN, Huie S, Li R-K, Morrison CJ, Warnock DW, Hajjeh RA: Candida dubliniensis fungemia: The first four cases in North America. Emerg Infect Dis 2000;6:46-49.

7 Meis JPGM, Ruhnke M, DePauw BE, Odds FC, Siegert W, Verweij PE: Candida dubliniensis candidemia in patients with chemotherapy-induced neutropenia and bone marrow transplantation. Emerg Infect Dis 1999;5:150_ 153.

-8 Sebti A, Kiehn TE, Perlin D, Chaturvedi V, Wong M, Doney A, Park S, Sepkowitz KA: Candida dubliniensis at a cancer center. Clin Infect Dis 2001;32:1034-1038.

-9 Coleman DC, Rinaldi MG, Haynes KA, Rex JH, Summerbell RC, Annaissie EA, Li A, Sullivan DJ: Importance of Candida species other than Candida albicans as opportunistic pathogen. Med Mycol 1998;36(suppl 1):156-165.

10 Khan ZU, Ahmad S, Mokaddas E, Chandy R: Simplified sunflower (Halianthus annuиs) seed agar for differentiation of Candida dubliniensis from Candida albicans. Clin Microbiol Infect 2004;10:590-592.

11 Staib P, Morschhauser J: Chlamydospore formation on Staib agar as a species-specific characteristic of Candida dubliniensis. Mycoses 1999;42:521-524.

-12 Al Mosaid A, Sullivan DJ, Coleman DC: Differentiation of Candida dubliniensis from Candida albicans on Pal's agar. J Clin Microbiol 2003;4:4787-4789.

13 Khan ZU, Ahmad S, Mokaddas E, Chandy R: Tobacco agar: A new medium for differentiation of Candida dubliniensis from Candida albicans. J Clin Microbiol 2004;42;4796-4798.

- 14 Mosca CO, Moragues MD, Llovo J, Al Mosaid A, Coleman DC, Ponton J: Casein agar: A useful medium for differentiating Candida dubliniensis from Candida albicans. J Clin Microbiol 2003;41:1259-1262.

15 Kirkpatrick WR, Revankar SG, Mcatee RK, Lopez-Ribot JL, Fothergill AW, McCarthy DI, Sanche SE, Cantu RA, Rinaldi MG, Patterson TF: Detection of Candida dubliniensis in oropharyngeal samples from human immunodeficiency virus-infected patients in North America by primary CHROMagar candida screening and susceptibility testing of isolates. J Clin Microbiol 1998;36:3007-3012.

16 Donnelly SM, Sullivan DJ, Shanley DB, Coleman DC: Phylogenetic analysis and rapid identification of Candida dubliniensis based on analysis of $A C T 1$ intron and exon sequences. Microbiology 1999;145:1871-1882.

17 Kurzai O, Heinz WJ, Sullivan DJ, Coleman DC, Frosch M, Muhlschlegel FA: Rapid PCR test for discriminating between Candida albicans and Candida dubliniensis isolates using primers derived from the $\mathrm{pH}$ regulated $P H R 1$ and PHR2 genes of C. albicans. J Clin Microbiol 1999;37:1587-1590.
8 Ahmad S, Khan Z, Mustafa AS, Khan ZU: Seminested PCR for diagnosis of candidemia: Comparison with culture, antigen detection, and biochemical methods for species identification. J Clin Microbiol 2002;40:2483-2489.

19 Ahmad S, Mustafa AS, Khan Z, Al-Rifaiy AI, Khan ZU: PCR-enzyme immunoassay of rDNA in the diagnosis of candidemia and comparison with amplicon detection by agarose gel electrophoresis. Int J Med Microbiol 2004; 294:45-51.

20 Ahmad S, Khan Z, Mokaddas E, Khan ZU: Isolation and molecular identification of $\mathrm{Can}$ dida dubliniensis from non-human immunodeficiency virus-infected patients in Kuwait. J Med Microbiol 2004;53:633-637.

21 McGinnis MR: Mycology; in Isenberg HD (ed): Clinical Microbiology Procedures Handbook. Washington, American Society for Microbiology Press, 1994, section 6, pp 6.1.1.6.1.12.

22 Alves SH, Milan EP, de Laet Sant'Ana P, Oliveira LO, Santurio JM, Colombo AL: Hypertonic sabouraud broth as a simple and powerful test for Candida dubliniensis screening. Diagn Mirobiol Infect Dis 2002;43:85-86.

23 Ahmad S, Mokaddas E, Al-Anoud J: Rapid detection of ethambutol-resistant Mycobacterium tuberculosis strains by PCR-RFLP targeting $e m b B$ codons 306 and 497 and iniA 501 mutations. Mol Cell Probes 2004; 18:299306.

24 National Committee for Clinical Laboratory Standards: Reference Method for Broth Dilution Antifungal Susceptibility Testing of Yeast, Approved Standard M27-A. Wayne, National Committee for Clinical Laboratory Standards, 1997.

25 Pfaller MA, Messer SA, Karlsson A, Bolmstrom A: Evaluation of the Etest method for determining fluconazole susceptibilities of 402 clinical yeast isolates by using three different agar media. J Clin Microbiol 1998;36:25862589.

26 Pfaller MA, Messer SA, Houston A, Mills K, Bolmstrom A, Jones RN: Evaluation of the Etest method for determining voriconazole susceptibilities of 312 clinical isolates of $\mathrm{Can}$ dida species by using three different agar media. J Clin Microbiol 2000;38:3715-3717.

27 Gales AC, Pfaller MA, Houston AK, Jolly SDJ, Sullivan DJ, Coleman DC, Soll DR: Identification of Candida dubliniensis based on temperature and utilization of xylose and alpha-methyl- $D$-glucoside as determined with the API 20C AUX and Vitek YBC Systems. J Clin Microbiol 1999;37:3804-3808.
28 Sancak B, Rex JH, Paetznick V, Chen E, Rodriguez J: Evaluation of a method for identification of Candida dubliniensis bloodstream isolates. J Clin Microbiol 2003;41:489-491.

29 Pincus DH, Coleman DC, Pruitt WR, Padhye AA, Salkin IF, Geimer M, Bassel A, Sullivan DJ, Clarke M, Hearn V: Rapid identification of Candida dubliniensis with commercial yeast identification systems. J Clin Microbiol 1999; 37:3533-3539.

- 30 Kurzai O, Korting HC, Harmsen D, Bautsch W, Molitor M, Frosch M, Muhlschlegel FA: Molecular and phenotypic identification of the yeast pathogen Candida dubliniensis. J Mol Med 2000; 78:521-529.

- 31 Tintelnot K, Haase G, Seibold M, Bergmann F, Staemmler M, Franz T, Naumann D: Evaluation of phenotypic markers for selection and identification of Candida dubliniensis. J Clin Microbiol 2000;38:1599-1608.

32 Gutierrez J, Morales P, Gonzalez MA, Quindos G: Candida dubliniensis, a new fungal pathogen. J Basic Microbiol 2002;42:207227.

- 33 Al-Hedaithy SS: The yeast species causing fungemia at a university hospital in Riyadh, Saudi Arabia, during a 10-year period. Mycoses 2003;46:293-298.

- 34 Polacheck I, Strahilevitz J, Sullivan D, Donnelly S, Salkin IF, Coleman DC: Recovery of Candida dubliniensis from non-human immunodeficiency virus-infected patients in Israel. J Clin Microbiol 2000;38:170-174.

35 Ponton J, Ruchel R, Clemons KV, Coleman DC, Grillot R, Guarro J, Aldebert D, Ambroise-Thomas P, Cano J, Carrillo-Munoz AJ, Gene J, Pinel C, Stevens DA, Sullivan DJ: Emerging pathogens. Med Mycol 2000;38 (suppl 1):225-236.

36 Lefler E, McCullough MJ, Clemons KV, Stevens DA: Initial isolation of Candida dubliniensis from the Middle East. Int J Infect Dis 2001;5:40-42.

- 37 Fotedar R, Al Hedaithy SS: Prevalence of Candida dubliniensis among germ tube-positive yeasts recovered from the respiratory specimens in HIV-negative patients. Mycoses 2004; 47:50-155.

38 Fotedar R, Al-Hedaithy SS: Candida dubliniensis at a university hospital in Saudi Arabia. J Clin Microbiol 2003;41:1907-1911.

39 Quindos G, Carrillo-Munoz AJ, Arevalo MP, Salgado J, Alonso-Vargas R, Rodrigo JM, Ruesga MT, Valverde A, Peman J, Canton E, Martin-Mazuelos E, Ponton J: In vitro susceptibility of Candida dubliniensis to current and new antifungal agents. Chemotherapy 2000; 46:395-401. 